\title{
OPERATORS WITH A STRICTLY CYCLIC VECTOR ${ }^{1}$
}

\author{
BRUCE BARNES
}

\begin{abstract}
In this paper, operators that have a strictly cyclic vector in a Banach space $X$ are studied. It is shown that the spectrum of such operators has some special properties. When $X$ is a Hilbert space, the connection between having a strictly cyclic vector and irreducibility is explored. If $X$ is an infinite dimensional Hilbert space, it is shown that no hyponormal operator on $X$ has a strictly cyclic vector.
\end{abstract}

1. Introduction. Throughout this paper, $X$ is a Banach space and $B(X)$ is the algebra of all bounded linear operators on $X$. If $T \in B(X)$, let $A(T)$ be the closed subalgebra of $B(X)$ generated by $T$ and $I$ (the identity operator on $X$ ). A vector $x_{0} \in X$ is strictly cyclic for $T$, if

$$
A(T) x_{0}=\left\{S x_{0}: S \in A(T)\right\}=X .
$$

If $T$ has a strictly cyclic vector in $X$, then we call $T$ strictly cyclic.

When $X=l^{p}, 1 \leqq p<+\infty$, several authors have studied strictly cyclic weighted shifts on $X$ ([2], [5], [6]). In this paper we consider the following question: what kind of operators can have a strictly cyclic vector in a given Banach space $X$ ? We find a partial answer to this question by investigating the class of strictly cyclic operators on $X$. When $X$ is reflexive, we show that strictly cyclic operators on $X$ have some very special properties. For example, when $H$ is an infinite dimensional Hilbert space and $T \in B(H)$ is strictly cyclic, then $T$ is similar to an irreducible operator [Proposition 7], the spectrum of $T^{*}$ consists entirely of eigenvalues [Corollary 3], and $T$ is not hyponormal [Theorem 8].

We mention that as a by-product of our investigation of strictly cyclic operators, we have some new results concerning strictly cyclic weighted shifts [Corollary 3] and [Proposition 9].

2. Basic properties. The following notation is in use throughout this paper: If $T \in B(X)$, then $\operatorname{sp}(T)$ denotes the spectrum of $T, \rho(T)$ denotes the spectral radius of $T$, and the range and null space of $T$ are denoted by $\mathscr{R}(T)$ and $\mathscr{N}(T)$, respectively.

Received by the editors March 20, 1973.

AMS (MOS) subject classifications (1970). Primary 47B99.

Key words and phrases. Strictly cyclic vector, strictly cyclic operator.

${ }^{1}$ This research was partially supported by NSF grant GP-28250.

(c) American Mathematical Society 1973 
In this section we prove some of the basic properties of strictly cyclic operators.

Proposition 1. Assume that $T \in B(X)$ has a strictly cyclic vector $x_{0} \in X$. Then

(1) the map $S \rightarrow S x_{0}$ is a bicontinuous isomorphism of $A(T)$ onto $X$, and

(2) if $S \in B(X)$ and $S$ commutes with $T$, then $S \in A(T)$.

In particular, (2) implies that $A(T)$ is weakly closed.

Proof. Assume $S \in B(X)$ and $S$ commutes with $T$. Since $A(T) x_{0}=X$, there exists $R \in A(T)$ such that $R x_{0}=S x_{0}$. Then $x_{0} \in \mathscr{N}(R-S)$, so that $X=A(T) x_{0} \subset \mathscr{N}(R-S)$. Therefore $S=R \in A(T)$. This same argument proves that $S \rightarrow S x_{0}$ is an isomorphism of $A(T)$ onto $X$, and since this map is continuous, it is bicontinuous by the closed graph theorem. This proves both (1) and (2).

Let $T \in B(X)$, and let $B$ be the weakly closed subalgebra of $B(X)$ generated by $T$ and $I$. Some authors call $x_{0} \in X$ a strictly cyclic vector for $T$ if $B x_{0}=X$. This definition is equivalent to the one we have used. For assume that $B x_{0}=X$. As in the proof of Proposition 1, one can establish that $S \rightarrow S x_{0}$ is a bicontinuous isomorphism of $B$ onto $X$. Therefore $A(T) x_{0}$ is a closed subspace of $X$. Then since $A(T)$ is weakly dense in $B$ and $B x_{0}=X$, it follows that $A x_{0}$ is weakly dense in $X$. Therefore $A x_{0}=X$, so that $x_{0}$ is a strictly cyclic vector for $T$.

If $x \in X$, let $\langle x\rangle$ denote the subspace of $X$ spanned by $x$.

THeOREM 2. Assume that $T \in B(X)$ has a strictly cyclic vector $x_{0} \in X$. Then $T$ is invertible if and only if $x_{0} \in(\mathscr{R}(T))^{-}$. Furthermore, if $T$ is not invertible, then $X=\left\langle x_{0}\right\rangle \oplus(\mathscr{R}(T))$ -

Proof. Suppose that $x_{0} \in(\mathscr{R}(T))^{-}$. Then there exists a sequence $\left\{y_{n}\right\} \subset X$ such that $T y_{n} \rightarrow x_{0}$. There exists a sequence of polynomials $Q_{n}(\lambda)$ such that $\left\|Q_{n}(T) x_{0}-y_{n}\right\| \rightarrow 0$. Therefore, $\left\|T Q_{n}(T) x_{0}-x_{0}\right\| \rightarrow 0$. Let $P_{n}(\lambda)=\lambda Q_{n}(\lambda)$. We have that $\left\|\left(P_{n}(T)-I\right) x_{0}\right\| \rightarrow 0$, so by Proposition 1(1), $\left\|P_{n}(T)-I\right\| \rightarrow 0$. This implies that there exists a positive integer $m$ such that $P_{m}(T)$ is invertible. Then, since $\operatorname{sp}\left(P_{m}(T)\right)=P_{m}(\operatorname{sp}(T))$ and $P_{m}(0)=0$, it follows that $0 \notin \mathrm{sp}(T)$. Thus when $x_{0} \in(\mathscr{R}(T))^{-}$, then $T$ is invertible. The converse is obvious.

Now assume that $T$ is not invertible. Then by the previous argument, $x_{0} \notin(\mathscr{R}(T))^{-}$. If $y_{0} \in X$, then there exists a sequence of polynomials $\left\{P_{n}(\lambda)\right\}$ such that $\left\|P_{n}(T) x_{0}-y_{0}\right\| \rightarrow 0$. Then since $P_{n}(T) x_{0} \in\left\langle x_{0}\right\rangle \oplus(\mathscr{R}(T))^{-}$ for all $n \geqq 1$ and $\left\langle x_{0}\right\rangle \oplus(\mathscr{R}(T))^{-}$is a closed subspace of $X$, it follows that $y_{0} \in\left\langle x_{0}\right\rangle \oplus(\mathscr{R}(T))^{-}$. This completes the proof of the theorem.

If $T \in B(X)$, let $T^{\prime}$ denote the adjoint operator of $T$. When $X$ is a Hilbert space, we use $T^{*}$ to denote the Hilbert space adjoint of $T$. 
COROllary 3. Assume that $T \in B(X)$ has a strictly cyclic vector $x_{0} \in X$. Then $\operatorname{sp}\left(T^{\prime}\right)$ (or $\operatorname{sp}\left(T^{*}\right)$, if $X$ is a Hilbert space) consists entirely of eigenvalues, and the corresponding eigenspaces are all one dimensional.

Proof. The vector $x_{0}$ is a strictly cyclic vector for $\lambda-T$ for any scalar $\lambda$. If $\lambda \in \operatorname{sp}\left(T^{\prime}\right)$ (or $\bar{\lambda} \in \mathrm{sp}\left(T^{*}\right)$, if $X$ is a Hilbert space), then $\lambda \in \operatorname{sp}(T)$, so that $\lambda-T$ is not invertible. Then by Theorem $2,(\mathscr{R}(\lambda-T))^{-}$has codimension one in $X$. This implies the corollary.

If $T \in B(X)$, then $A(T)$ is a commutative Banach algebra. Let $\Omega$ be the carrier space of $A(T)$, i.e. $\Omega$ is the set of all nonzero multiplicative linear functionals on $A(T)$. If $S \in A(T)$, then we denote by $S$ (the Gel'fand transform of $S$ ) the function defined on $\Omega$ by $S(\omega)=\omega(S), \omega \in \Omega$. We use this notation in the next theorem.

THEOREM 4. Assume that $X$ is reflexive and that $T \in B(X)$ is strictly cyclic. If $\lambda_{0} \in \operatorname{sp}(T)$ and $\left|\lambda_{0}\right|=\|T\|$, then $\lambda_{0}$ is an isolated point of $\operatorname{sp}(T)$.

Proof. First we note that by Proposition 1(2), the spectrum of $T$ relative to the commutative Banach algebra $A(T)$ is the same as $\operatorname{sp}(T)$. We may assume $\lambda_{0} \neq 0$. Let $\Omega$ be the carrier space of $A(T)$. Define

$$
\Gamma_{0}=\left\{\omega \in \Omega: \hat{T}(\omega)=\lambda_{0}\right\} .
$$

Let $S=\frac{1}{2}\left(\lambda_{0}^{-1} T+I\right)$. Then by the proof of [7, Lemma (3.3.10), p. 138], $\|S\|=1$ and $\{\omega \in \Omega: \hat{S}(\omega)=1\}=\{\omega \in \Omega:|\hat{S}(\omega)|=1\}=\Gamma_{0}$. Thus $\hat{S}(\omega)=1$ for $\omega \in \Gamma_{0},|\hat{S}(\omega)|<1$ for $\omega \notin \Gamma_{0}$, and $\left\{S^{n}\right\}$ is a bounded sequence in $A(T)$. Now by Proposition 1, $A(T)$ is bicontinuously isomorphic to the reflexive Banach space $X$. It follows that any bounded set in $A(T)$ is weakly sequentially compact $[1$, Theorem 28, p. 68]. Therefore some subsequence of $\left\{S^{n}\right\}$ converges weakly to an element $R \in A(T)$. Since every $\omega \in \Omega$ is a continuous linear functional on $A(T)$, then $\hat{R}(\omega)=1$ if $\omega \in \Gamma_{0}$ and $\hat{R}(\omega)=0$ if $\omega \notin \Gamma_{0}$. Let $V=T-\lambda_{0} R$. Then

$$
\operatorname{sp}(V)=\{\hat{V}(\omega): \omega \in \Omega\}=\left(\operatorname{sp}(T) \backslash\left\{\lambda_{0}\right\}\right) \cup\{0\} .
$$

Since $\operatorname{sp}(T)$ and $\operatorname{sp}(V)$ are compact sets, $\left\{\lambda_{0}\right\}$ is an isolated point of $\operatorname{sp}(T)$.

3. Irreducibility. In this section we prove that if $T \in B(H)$ is strictly cyclic, then $T$ is the orthogonal direct sum of a finite number of irreducible operators [Theorem 6] and $T$ is similar to an irreducible operator [Proposition 7]. But first, we give an example of a reducible, strictly cyclic operator on an infinite dimensional Hilbert space.

EXAMPLe 5. Assume that $H$ is an infinite dimensional Hilbert space. Fix a positive integer $n$. Let $\left\{x_{1}, \cdots, x_{n}\right\}$ be an orthonormal set in $H$. Let $E_{k}$ be the orthogonal projection of $H$ onto $\left\langle x_{k}\right\rangle, 1 \leqq k \leqq n$. Let 
$K=\left\{x_{1}, \cdots, x_{n}\right\}^{\perp}$. Choose positive real numbers, $\lambda_{k}, 1 \leqq k \leqq n+1$, such that $\lambda_{1}>\lambda_{2}>\cdots>\lambda_{n+1}$. Choose $S \in B(H)$ such that $S\left(x_{k}\right)=0$ for $1 \leqq k \leqq n, S(K) \subset K,\left.S\right|_{K}$ has a strictly cyclic vector $x_{n+1} \in K$, and $\lambda_{n+1}>\|S\|$. Let $T=\lambda_{1} E_{1}+\cdots+\lambda_{n} E_{n}+S$. Then

$$
\left(\lambda_{1}^{-1} T\right)^{m}=E_{1}+\left(\lambda_{1}^{-1} \lambda_{2}\right)^{m} E_{2}+\cdots+\left(\lambda_{1}^{-1} \lambda_{n}\right)^{m} E_{n}+\left(\lambda_{1}^{-1} S\right)^{m} .
$$

Thus $\left(\lambda_{1}^{-1} T\right)^{m} \rightarrow E_{1}$ as $m \rightarrow+\infty$. Then $E_{1} \in A(T)$. Let $T_{1}=\lambda_{2} E_{2}+\cdots+$ $\lambda_{n} E_{n}+S \in A(T)$. The same argument as before proves that $\left(\lambda_{2}^{-1} T_{1}\right)^{m} \rightarrow E_{2}$ as $m \rightarrow+\infty$. Therefore $E_{2} \in A(T)$, and repeating this argument we have $E_{k} \in A(T), 1 \leqq k \leqq n$, and $S \in A(T)$.

Now let $x_{0}=x_{1}+\cdots+x_{n+1}$. We proceed to verify that $x_{0}$ is a strictly cyclic vector for $T$. Let $y_{0} \in H$ be given. Then $y_{0}=\mu_{1} x_{1}+\cdots+\mu_{n} x_{n}+z_{0}$ where the $\mu_{k}$ are scalars and $z_{0} \in K$. Let $I_{K}=I-\left(E_{1}+\cdots+E_{n}\right) \in A(T)$. Since $x_{n+1} \in K$ is strictly cyclic for $\left.S\right|_{K}$, then there exists $R$ in the closed subalgebra of $B(H)$ generated by $I_{K}$ and $S$ such that $R\left(x_{n+1}\right)=z_{0}$. Note that $R \in A(T)$ and $R\left(x_{k}\right)=0,1 \leqq k \leqq n$. Then $W=\mu_{1} E_{1}+\cdots+\mu_{n} E_{n}+$ $R \in A(T)$, and $W x_{0}=y_{0}$. Thus $x_{0}$ is strictly cyclic for $T$.

THEOREM 6. Assume that $T \in B(H)$ has a strictly cyclic vector $x_{0} \in H$. Then there exists a finite mutually orthogonal collection of subspaces of $H$ that reduce $T,\left\{H_{1}, \cdots, H_{n}\right\}$, such that $H=H_{1} \oplus \cdots \oplus H_{n}$ and $T=T_{1} \oplus \cdots \oplus T_{n}$ where each $T_{k}$ is an irreducible operator in $B\left(H_{k}\right)$ with a strictly cyclic vector in $H_{k}$.

Proof. Let $B$ be the closed *-subalgebra of $B(H)$ generated by $T$, $T^{*}$, and $I$. Define a positive functional $f$ on $B$ by $f(S)=\left(S x_{0}, x_{0}\right), S \in B$. Let

$$
K_{f}=\left\{S \in B: f\left(S^{*} S\right)=0\right\}=\left\{S \in B: S x_{0}=0\right\} .
$$

The quotient space $B-K_{f}=\left\{S+K_{f}: S \in B\right\}$ is normed by the norm

$$
\left\|S+K_{f}\right\|_{f}=f\left(S^{*} S\right)^{1 / 2}=\left\|S x_{0}\right\|,
$$

and by the usual quotient norm,

$$
\left\|S+K_{f}\right\|=\inf \left\{\|S-R\|: R \in K_{f}\right\} .
$$

It is easy to verify that $B-K_{f}$ is complete in both these norms and that $\left\|x_{0}\right\|\left\|S+K_{f}\right\| \geqq\left\|S+K_{f}\right\|_{f}$ for all $S \in B$. Then by the closed graph theorem the norms are equivalent on $B-K_{f}$. Certain of the arguments of $\mathrm{H}$. Halpern in [4] apply exactly to this situation. The commutant $B^{\prime}$ of $B$ in $B(H)$ has the properties:

(i) If $F$ is a nonzero projection in $B^{\prime}$, then $F$ majorizes a minimal projection $E \in B^{\prime}$ [4, Lemma 1].

(ii) A maximal set of mutually orthogonal minimal projections in $B^{\prime}$ must be finite [4, the proof of Proposition 2]. 
Now by (i) we can choose a nonempty maximal set of mutually orthogonal minimal projections in $B^{\prime}$, and by (ii), this set is finite. Let $\left\{E_{1}, \cdots\right.$, $\left.E_{n}\right\}$ be this set, and let $H_{k}=\mathscr{R}\left(E_{k}\right), 1 \leqq k \leqq n$. Then $H$ is the orthogonal direct sum of the subspaces $H_{k}$, the collection $\left\{H_{1}, \cdots, H_{n}\right\}$ reduces $B$, and $B$ acts irreducibly on each $H_{k}, 1 \leqq k \leqq n$. Write $x_{0}$ as $x_{1}+\cdots+x_{n}$ where $x_{k} \in H_{k}, 1 \leqq k \leqq n$, and let $T_{k}=\left.T\right|_{H_{k}}, 1 \leqq k \leqq n$. Then $T=T_{1} \oplus \cdots \oplus$ $T_{n}$. If $y \in H_{k}$, then for some $R \in A(T)$,

$$
R x_{0}=R x_{k}=\left(\left.R\right|_{H_{k}}\right) x_{k}=y .
$$

There exists a sequence of polynomials $\left\{P_{n}(\lambda)\right\}$ such that $P_{n}(T) \rightarrow R$. Therefore

$$
P_{n}\left(\left.T\right|_{H_{k}}\right)=\left.\left.P_{n}(T)\right|_{H_{k}} \rightarrow R\right|_{H_{k}} .
$$

Then $\left.R\right|_{H_{k}} \in A\left(T_{k}\right)$ and $\left(\left.R\right|_{H_{k}}\right) x_{k}=y$ : This proves that $x_{k}$ is strictly cyclic for $T_{k}$ on $H_{k}$.

Proposition 7. If $T \in B(H)$ has a strictly cyclic vector $x_{0} \in H$, then $T$ is similar to an irreducible operator.

Proof. Choose $\lambda \in \operatorname{sp}(T)$. By Theorem 2, $H=\left\langle x_{0}\right\rangle \oplus(\mathscr{R}(\lambda I-T))^{-}$. Choose $V$ an invertible operator in $B(H)$ such that $V x_{0}=x_{0}$ and $V$ maps $\left\langle x_{0}\right\rangle^{\perp}$ onto $(\mathscr{R}(\lambda I-T))^{-}$. Let $S=V^{-1}(\lambda I-T) V=\lambda I-V^{-1} T V$. Then $x_{0}$ is strictly cyclic for $S$ and $\left\langle x_{0}\right\rangle=\mathscr{N}\left(S^{*}\right)=(\mathscr{R}(S))^{-\perp}$. Then the argument used in [3, p. 895] to prove that weighted shifts are irreducible applies to $S$. Then since $S$ is irreducible, $V^{-1} T V$ is irreducible.

4. Some operators that are not strictly cyclic. The results of $\S \S 2$ and 3 point to the conclusion that strictly cyclic operators on a reflexive space are very special. In this section we add further evidence pointing in the same direction by showing that no hyponormal operator on an infinite dimensional Hilbert space is strictly cyclic. Also, later in this section, we prove that any weighted shift $S$ on $l^{p}, 1<p<+\infty$, such that $\|S\|=\rho(S)$, is not strictly cyclic.

THEOREM 8. Assume that $T$ is a hyponormal operator on an infinite dimensional Hilbert space $H$. Then $T$ does not have a strictly cyclic vector in $H$.

Proof. Assume that $T$ has a strictly cyclic vector in $H$. Then by Proposition $1, A(T)$ is a reflexive Banach space. Suppose that a scalar $\lambda_{1}$ is an eigenvalue for $T$. Let $E$ be the projection with range $\mathscr{N}\left(\lambda_{1} I-T\right)$. Then $T E=\lambda_{1} E$, and by [10, Lemma 2], $T^{*} E=\bar{\lambda}_{1} E$. Therefore $E T=T E=\lambda_{1} E$. By Proposition 1 it follows that $E \in A(T)$. Suppose that $T$ has an infinite 
sequence $\left\{\lambda_{k}\right\}$ of distinct eigenvalues and let $\left\{E_{k}\right\}$ be the corresponding sequence of projections such that $E_{k} T=T E_{k}=\lambda_{k} E_{k}$. By [10, Lemma 3], $E_{k} E_{j}=0$ if $k \neq j$. Then the sequence of projections generates a closed commutative *-subalgebra $C$ of $B(H)$ such that $C \subset A(T)$. Thus $C$ is a reflexive Banach space. Since $C$ is infinite dimensional, this contradicts a result of S. Sakai [9, Proposition 2]. Thus $T$ has at most a finite number of distinct eigenvalues.

Now by [10, Theorem 1], $\|T\|=\rho(T)$. If $\lambda_{0} \in \operatorname{sp}(T)$ and $\left|\lambda_{0}\right|=\|T\|$, then by Theorem $4, \lambda_{0}$ is an isolated point of the spectrum of $T$. Then by [10, Theorem 2], $\lambda_{0}$ is an eigenvalue of $T$. Therefore $T$ has an eigenvalue. Let $\left\{\lambda_{0}, \cdots, \lambda_{n}\right\}$ be the collection of all the distinct eigenvalues of $T$ (this collection must be finite). Let $\left\{E_{0}, \cdots, E_{n}\right\}$ be the corresponding projections such that $T E_{k}=E_{k} T=\lambda_{k} T, 0 \leqq k \leqq n$, and $E_{k} E_{j}=0, k \neq j$. Let $N=\sum_{k=0}^{n} \lambda_{k} E_{k} \in A(T)$. Since $N$ is normal and $N$ commutes with $T$, it is easy to verify that $T-N$ is hyponormal. Then, arguing as before, there must exist an eigenvalue $\mu_{0}$ of $T-N$ such that $\left|\mu_{0}\right|=\|T-N\|$. Assume $\mu_{0} \neq 0$. Choose $y \in H, y \neq 0$, such that $(T-N) y=\mu_{0} y$. Then $E_{k}\left(\mu_{0} y\right)=E_{k}(T-N) y=0,0 \leqq k \leqq n$. Therefore $N(y)=\sum_{k=0}^{n} \lambda_{k} E_{k} y=0$. It follows that $T y=(T-N) y=\mu_{0} y$. Then $\mu_{0}=\lambda_{k}$ for some $k$, which is a contradiction since $E_{k} y=0$. Thus $\|T-N\|=\left|\mu_{0}\right|=0$, and this is clearly impossible since $H$ is infinite dimensional.

Assume that $H$ is an infinite dimensional Hilbert space and $T \in B(H)$ is hyponormal. If $T^{*}$ is strictly cyclic, then $A\left(T^{*}\right)$ is a reflexive Banach space. It follows that $A(T)$ is reflexive. This, as in the proof of Theorem 8 , leads to a contradiction. Thus the adjoint of a hyponormal operator on $H$ cannot be strictly cyclic.

Proposition 9. If $S$ is a strictly cyclic weighted shift on $l^{p}, 1<p<+\infty$, then $\rho(S)<\|S\|$.

Proof. Assume that $S$ is a strictly cyclic shift on $l^{p}$ for some $p$, $1<p<+\infty$, and $\rho(S)=\|S\|=1$. Choose $\lambda_{0} \in \operatorname{sp}(S)$ such that $\left|\lambda_{0}\right|=1$. By Theorem $4, \lambda_{0}$ is an isolated point of $\operatorname{sp}(S)$. But $\operatorname{sp}(S)$ is a disk centered at 0 [8, Corollary, p. 351]. This is a contradiction, since $\lambda_{0}$ is an isolated point of $\operatorname{sp}(S)$.

Let $\left\{e_{n}\right\}_{n=1}^{+\infty}$ denote the standard Schauder basis in any of the $l^{p}$ spaces, $1 \leqq p<+\infty$. Let $\left\{\alpha_{k}\right\}$ be a sequence of nonzero scalars, and let $S$ be the weighted shift on $l^{p}$ such that $S e_{n}=\alpha_{n} e_{n+1}, n \geqq 1$. Making use of an argument due to J. Stampfli [11], we prove that if $\lim \left|\alpha_{n}\right|=\|S\|$, then $\rho(S)=\|S\|$. Thus by Proposition 9, no weighted shift $S$ on $l^{p}, 1<p<+\infty$, with the property that $\lim \left|\alpha_{n}\right|=\|S\|$ is strictly cyclic.

We assume for convenience that $\|S\|=1$. Let $r ; t$ be any two real numbers such that $0<r<t<1$. Since $\lim \left|\alpha_{n}\right|=1$, then $r\left|\alpha_{n}\right|^{-1}<t$ for all 
but a finite number of positive integers $n$. Set $\alpha_{0}=1$, and assume $p^{-1}+$ $q^{-1}=1$. Then the vector

$$
u=\sum_{n=1}^{+\infty}\left(\alpha_{0} \alpha_{1} \cdots \alpha_{n-1}\right)^{-1} r^{n} e_{n}
$$

is in $l^{a}$. A direct calculation verifies that $S^{\prime} u=r u\left(S^{\prime}\right.$ is the adjoint of $\left.S\right)$. Thus $\rho(S)=\rho\left(S^{\prime}\right) \geqq r$. This proves that $\rho(S)=1=\|S\|$.

ADDED IN PROOF. There is some overlap of $\S \S 2$ and 4 of this paper with two papers by Alan Lambert, [Pacific J. Math. 39 (1971), 717-726] and [Indiana Math. J. 22 (1973), 959-963]. The paper in the Indiana Math J. had not yet appeared when this article was submitted, and the author was unaware until recently of the paper in the Pacific J. Math.

\section{REFERENCES}

1. N. Dunford and J. Schwartz, Linear operators. I. General theory, Pure and Appl. Math., vol. 7, Interscience, New York, 1958. MR 22 \#8302.

2. M. Embry, Strictly cyclic operator algebras on a Banach space, Pacific J. Math. 45 (1973), 443-452.

3. P. R. Halmos, Ten problems in Hilbert space, Bull. Amer. Math. Soc. 76 (1970), 887-933. MR 42 \#5066.

4. H. Halpern, Finite sums of irreducible functionals on $C^{*}$-algebras, Proc. Amer. Math. Soc. 18 (1967), 352-358. MR 34 \#6551.

5. E. Kerlin and A. Lambert, Strictly cyclic shifts on $l_{p}$ (preprint).

6. A. Lambert, Strictly cyclic weighted shifts, Proc. Amer. Math. Soc. 29 (1971), 331-336. MR 43 \#970.

7. C. E. Rickart, General theory of Banach algebras, University Series in Higher Math., Van Nostrand, Princeton, N.J., 1960. MR 22 \#5903.

8. W. Ridge, Approximate point spectrum of a weighted shift, Trans. Amer. Math. Soc. 147 (1970), 349-356. MR 40 \#7843.

9. S. Sakai, Weakly compact operators on operator algebras, Pacific J. Math. 14 (1964), 659-664. MR 29 \#488.

10. J. G. Stampfli, Hyponormal operators, Pacific J. Math. 12 (1962), 1453-1458. MR 26 \#6772.

11. - Which weighted shifts are subnormal, Pacific J. Math. 17 (1966), 367-379. MR 33 \#1740.

Department of Mathematics, University of Oregon, Eugene, Oregon 97403

Current address: Department of Mathematics, University of Stirling, Stirling, Scotland 